\title{
Malformaciones vasculares del cuero cabelludo
}

Vascular malformations of the scalp

\author{
Ana María Toro', Juliana Santos², Delsy Yurledy Del Río3 \\ 1. Médica de planta, Hospital Pablo Tobón Uribe, Medellín, Colombia \\ 2. Médica dermatóloga, Universidad CES, Medellín, Colombia \\ 3. Médica dermatóloga, Hospital Pablo Tobón Uribe, Medellín, Colombia
}

\section{RESUMEN}

Las malformaciones arterio-venosas del cuero cabelludo son infrecuentes y de muy rara presentación en la población pediátrica. Consisten en comunicaciones anormales entre los vasos de la grasa subcutánea y las arterias nutricias que abastecen el cuero cabelludo. Su etiología puede ser congénita, traumática o idiopática. Clínicamente producen desde lesiones asintomáticas, molestia local o cefalea, hasta necrosis y hemorragia masiva.

El diagnóstico de esta entidad es clínico e imaginológico, y su tendencia es al crecimiento progresivo.

El tratamiento de elección es la resección quirúrgica extensa de toda la malformación y todas sus ramas nutricias, pues se observa recurrencia cuando no se elimina todo el aporte sanguíneo.

Se presenta el caso de un paciente con diagnóstico de malformación arterio-venosa en cuero cabelludo, de probable origen congénito, sin compromiso intracraneano y con adecuada reacción al manejo intravascular.

PALABRAS ClAVE: malformaciones arterio-venosas; cuero cabelludo; procedimientos intravasculares; niño.

\section{SUMMARY}

Scalp arteriovenous malformations are infrequent and very rare in the pediatric population. They consist of abnormal vessel communications between subcutaneous fatty layer vessels and the feeding arteries that supply the scalp. Its etiology might be congenital, traumatic or idiopathic. Clinically they might be asymptomatic or present with local discomfort, headache, and even necrosis and massive hemorrhage.

Diagnosis of this entity is made based on clinical findings and image exams and the tendency of the lesion is progressive growth.

The first line of treatment is extensive surgical resection of the entire malformation and its feeding arterial branches, since recurrences have been reported in cases where the blood supply was not removed entirely.

\author{
Correspondencia: \\ Ana María Toro \\ Email: \\ atorocadavid@gmail.com \\ Recibido: $12 / 12 / 2016$ \\ Aceptado: 08/05/2017
}

Conflictos de interés:

No se reportan conflictos de interés.

Financiación:

Ninguna. 
We report the case of a patient with arteriovenous malformation of the scalp, which may be congenital, without intracranial involvement and adequate response to endovascular treatment.

KEY WORDS: arteriovenous malformations; scalp; endovascular procedures; child.

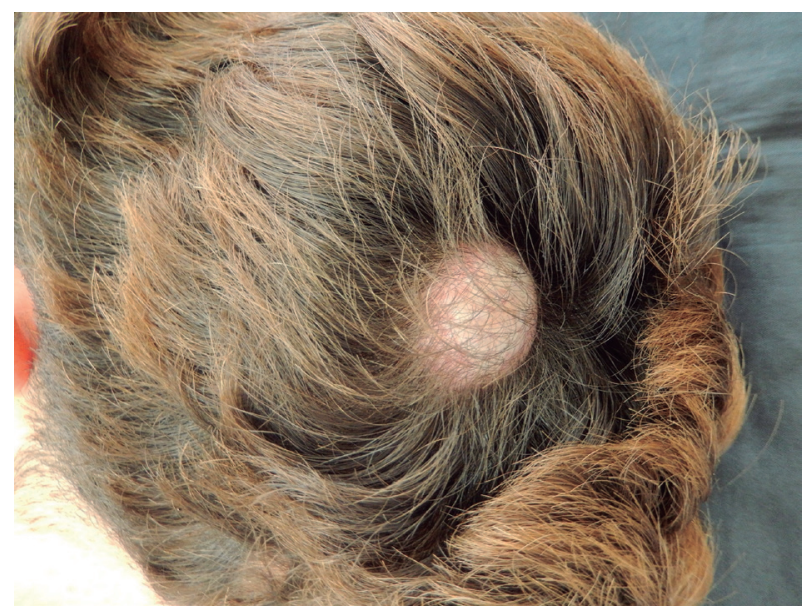

FIGURA 1. Masa en la región témporo-parietal derecha, de 4 x 3 $\mathrm{cm}$, aproximadamente, pulsátil, blanda y superficie con vasos sanguíneos.

\section{REPORTE DE CASO}

Se presenta un paciente de sexo masculino de 14 años de edad, residente en Guatapé (Antioquia), evaluado por el Servicio de Dermatología por una lesión nodular en el cuero cabelludo de cuatro años de evolución, pruriginosa, pulsátil, aproximadamente de $4 \times 3 \mathrm{~cm}$, con aumento progresivo de su tamaño, y localizada en la región témporo-parietal derecha (figura 1). Se hospitalizó con la impresión diagnóstica de malformación vascular, para la práctica de imágenes diagnósticas y manejo multidisciplinario.

En la resonancia magnética se reportó una lesión epicraneana, de $30 \times 21 \times 20 \mathrm{~mm}$, que comprometía el cuero cabelludo, con múltiples lesiones serpiginosas en su interior con vacío de señal, que sugiere estructuras vasculares, secundaria a una malformación arterio-venosa, alimentada por ramas de la arteria carótida externa derecha (arteria occipital, auricular posterior y temporal superficial), con drenaje al sistema venoso del cuero cabelludo. No se observó comunicación intracraneana.

Fue valorado por el Servicio de Neurointervencionismo, se le practicó una panangiografía cerebral y se sometió a tratamiento intravascular extracraneano, sin complicaciones. En la panangiografía cerebral posoperatoria de control se evidenció exclusión completa del nido de la malformación (figura 2, A y B).
La evolución clínica fue adecuada (figura 3), por lo que se dio de alta para seguimiento ambulatorio.

\section{DISCUSIÓN}

Las malformaciones arterio-venosas del cuero cabelludo son conexiones fistulosas anormales entre las arterias nutricias y las venas de drenaje, sin compromiso del lecho capilar del tejido subcutáneo ${ }^{(1)}$.

Fueron descritas por primera vez por Hunter en 1757 $\mathrm{y}$, actualmente, se conocen con diferentes nombres, como aneurismas serpentinum, aneurismas arteriovenosos, fístulas arterio-venosas, aneurismas racemosos, angiomas plexiformes o malformaciones arterio-venosas ${ }^{(2,3,4)}$.

Las ramas arteriales que nutren la malformación proceden, generalmente, de la arteria carótida externa, como en el presente caso, y, en algunas situaciones, de ramas de la arteria carótida interna ${ }^{(2)}$.

Según datos obtenidos de autopsias, la frecuencia de malformaciones arterio-venosas del cuero cabelludo es de 4,3\% en la población general ${ }^{(3)}$.

En la población pediátrica, las malformaciones arterio-venosas del cuero cabelludo ocurren con escasa frecuencia y difieren de las del adulto en cuanto a etiología, edad de presentación, impacto estético y excelente resultado con el tratamiento temprano ${ }^{(5)}$. 
Respecto a la etiología de estas lesiones, los datos son controversiales; sin embargo, se sabe que las malformaciones arterio-venosas pueden ser de origen congénito, traumático o idiopático. Estas últimas son significativamente más comunes y se han propuesto tres posibles hipótesis para explicar su etiología: la primera es la persistencia de comunicaciones arterio-venosas primitivas y agenesia de capilares; la segunda sugiere que las malformaciones arterio-venosas se originan de hamartomas vasculares, y la tercera las atribuye a la formación de una fístula en el sitio del cruce arteriovenoso $^{(3,6)}$.

Las malformaciones arterio-venosas de origen congénito o espontáneo pueden estar presentes al nacimiento, pero la mayoría de los recién nacidos son asintomáticos y las lesiones se manifiestan generalmente en la segunda década de la vida. Factores como el trauma, el embarazo, o los cambios hormonales pueden causar empeoramiento de los síntomas ${ }^{(1,5)}$. Generalmente, se localizan en las regiones frontal, temporal o parietal, y sus ramas nutricias son, en orden de frecuencia, la arteria temporal superficial, la occipital y la auricular posterior ${ }^{(2,6)}$.

Cerca de 10 a $20 \%$ de las malformaciones arterio-venosas del cuero cabelludo son de origen traumático y, en este caso, el curso de la arteria superficial temporal es generalmente el más comprometido; estas pueden ocurrir a cualquier edad y son el resultado de la comunicación directa entre arteria y vena, o de la canalización de trombos. Muchas lesiones de origen traumático se reportan después de trasplantes de cabello, de intervenciones, como craneotomías, o de infusiones a través de venas del cuero cabelludo. Su crecimiento es lento y son relevantes en un período de 6 a 12 años.
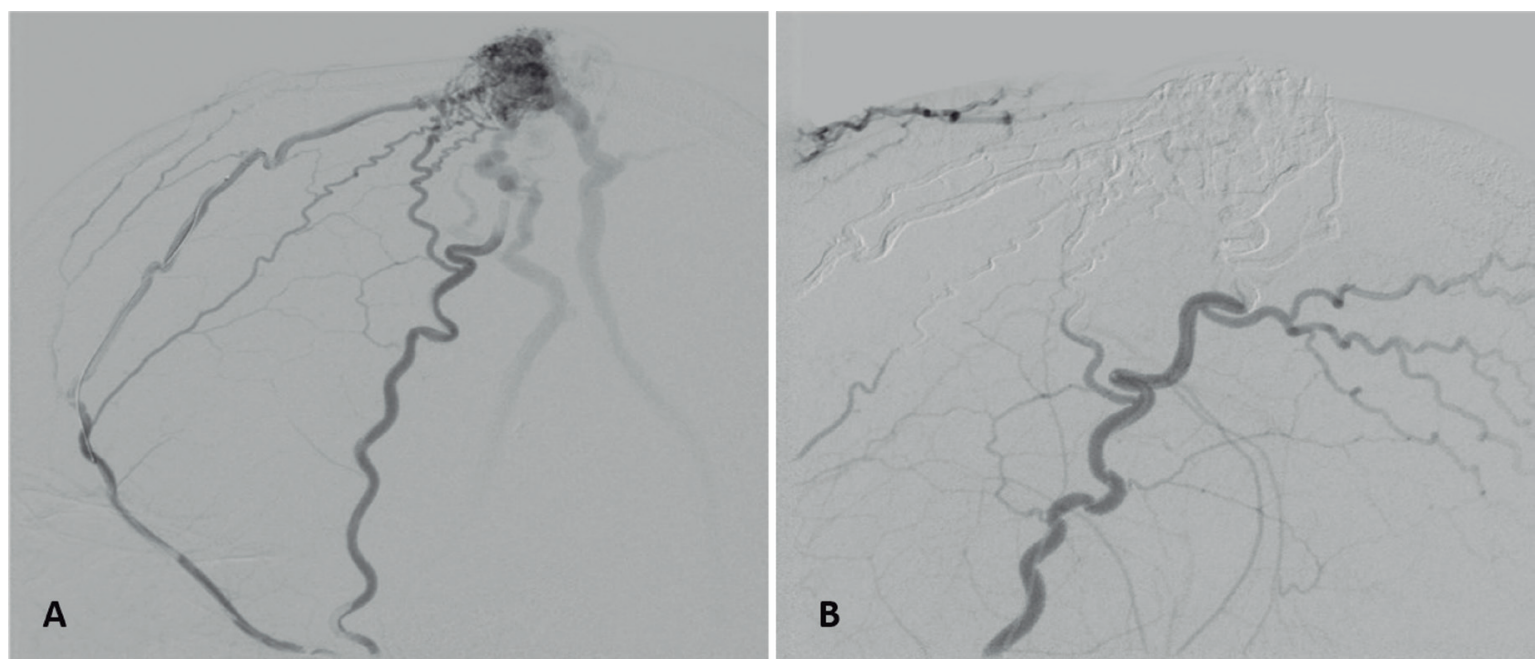

FIGURA 2. Panangiografía cerebral.

(A) Imagen durante procedimiento de producción de embolia, en la que se observa el nido de la malformación con drenaje venoso superficial.

(B) Imagen posterior al tratamiento intravascular en la que se evidencia la exclusión de la malformación arterio-venosa.

Las manifestaciones clínicas son diversas. Se pueden presentar como una tumoración subcutánea inocua en el cuero cabelludo o como una masa grande, grotesca y pulsátil. Pueden ser lesiones asintomáticas, o causar molestia local, cefalea, alopecia, ulceración de la piel, necrosis, crisis convulsivas, retardo psicomotor, falla cardiaca por alto flujo o hemorragia masiva. Otros síntomas específicos, según la localización, son acúfenos, alteraciones visuales y epistaxis ${ }^{(5,7)}$.

El diagnóstico de las malformaciones arterio-venosas es clínico e imaginológico, y se deben descartar diagnósticos diferenciales, como sinus pericranii (comuni- cación anormal entre el sistema venoso extracraneal y los senos venosos durales), encefalocele, hamartomas vasculares, hemangiomas y cavernomas, mediante ecografía Doppler, angiografía, resonancia magnética o angiografía intrarterial por substracción digital; esta última es el método de referencia ${ }^{(2,4)}$.

El tratamiento de elección es la resección quirúrgica extensa de toda la malformación y de todas sus ramas nutricias, debido a la posibilidad de recurrencia cuando no se elimina todo el aporte sanguíneo ${ }^{(2)}$. El manejo intravascular también es una opción como tratamiento definitivo o junto con la cirugía para re- 


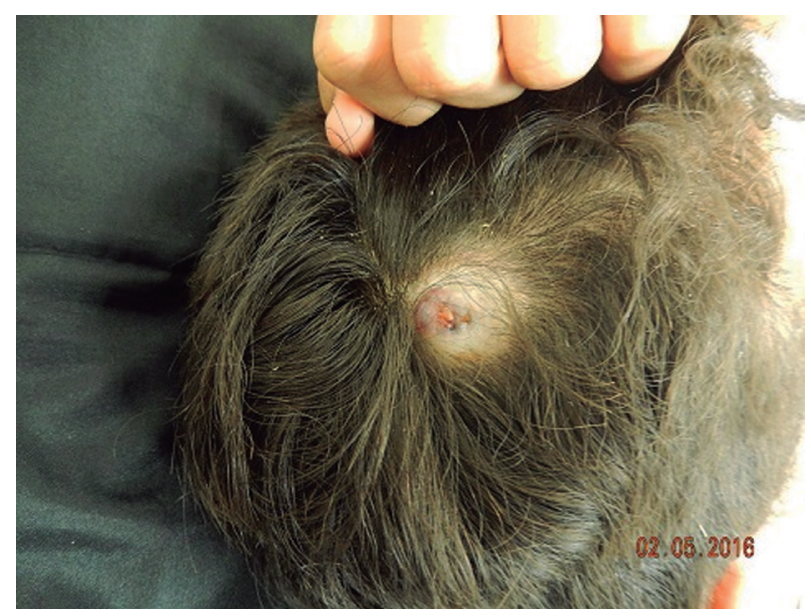

FIGURA 3. Lesión con una adecuada evolución clínica después del tratamiento intravascular extracraneano

ducir la extensión y el sangrado durante la resección; igualmente, está indicado para el control de hemorragia aguda.

La causa más común de falla en el tratamiento, incluso combinando las dos técnicas, es la resección incompleta $^{(7,8)}$.

Otras opciones de tratamiento incluyen la ligadura de los vasos nutricios y la inyección de agentes esclerosantes.

La elección de la modalidad del tratamiento depende del sitio, el tamaño y el patrón de drenaje de las malformaciones arterio-venosas; también, influyen la preferencia del paciente, la experiencia del médico tratante y los equipos disponibles ${ }^{(3,4)}$.

\section{CONCLUSIONES}

Se presenta el caso de un paciente con malformación arterio-venosa del cuero cabelludo, sin compromiso intracraneal, como una entidad poco frecuente en la población pediátrica.

Se resalta la importancia de hacer un diagnóstico clínico e imaginológico precoz, al igual que un tratamiento oportuno de esta entidad, combinando técnicas intravasculares y quirúrgicas, con el fin de minimizar el riesgo de sangrado, teniendo en cuenta la tendencia de estas lesiones al crecimiento progresivo, y el riesgo de complicaciones que pueden reflejarse en manifestaciones neurológicas y cardiovasculares.

Ante la sospecha clínica de una malformación arteriovenosa del cuero cabelludo, se deben descartar diagnósticos diferenciales como los tumores extracra- neales, mediante técnicas imaginológicas.

Al practicar el tratamiento quirúrgico, se debe resecar toda la lesión y sus ramas nutricias, para evitar recurrencias.

\section{REFERENCIAS}

1. Hasturk A, Erten F, Ayata T. Giant non-traumatic arteriovenous malformation of the scalp. Asian J Neurosurg. 2012;7:39.

2. García-Conde M, Martín-Viota L, Febles-García P, Cortés-Franco S. Malformación arteriovenosa gigante de cuero cabelludo: caso clínico. Neurocirugía. 2006;17:445-9.

3. Kumar R, Sharma G, Sharma BS. Management of scalp arterio-venous malformation: Case series and review of literature. Br J Neurosurg. 2012;26:371-7.

4. Kalyani R. Scalp arteriovenous malformation - A rare case. Sch J Appl Med Sci. 2013;1:441-3.

5. Gupta R, Kayal A. Scalp arteriovenous malformations in young. J Pediatr Neurosci. 2014;9:263.

6. Shenoy SN, Raja A. Scalp arteriovenous malformations. Neurol India. 2004;52:478-81.

7. Dmytriw AA, terBrugge KG, Krings T, Agid R. Endovascular treatment of head and neck arteriovenous malformations. Neuroradiology. 2014;56:22736.

8. Wan Najwa Zaini WM, Noreen Norfaraheen LA, Ahmad Sobri M. Scalp arteriovenous malformation: A case report. Malays J Med Sci. 2008;15:55-7. 